\title{
Efecto de acciones de equipos de Atención Primaria de Salud para proteger la salud mental después de desastres naturales
}

\author{
Effect of Primary Health Care team actions to protect \\ mental health after natural disasters
}

\author{
Alberto Minoletti ${ }^{1}$, Rubén Alvarado1, Verónica Vitriol2,3, Paula Sierralta1, \\ Camilo Bass ${ }^{4}$, Jorge Calvo ${ }^{3}$
}

\begin{abstract}
Resumen
Introducción: Existe alta prevalencia de alteraciones emocionales en la población expuesta a desastres y los centros de atención primaria de salud (APS) podrían contribuir a su prevención. Objetivo: Analizar asociaciones post-catástrofe entre variables de APS y variables de salud mental de la población. Método: Estudio efectuado en Chile un año después de un terremoto de $8,8^{\circ}$ Richter. Se aplicó un cuestionario de intensidad de acciones de salud mental en 16 centros APS y las escalas GHQ-12 y SF-36 para malestar psicológico y calidad de vida en una muestra basada en hogares y otra de consultantes a APS. Se efectuó un análisis de regresión lineal múltiple para las dos poblaciones y los dos instrumentos. Resultados: Ninguna de las variables sobre intensidad de acciones de APS mostró relación con GHQ-12 o SF-36 en ambas muestras. Las variables asociadas significativamente a mayor puntaje en GHQ-12 y menor puntaje en SF-36 fueron sexo femenino, baja escolaridad, baja situación económica, menor apoyo social y daños producidos por el terremoto. Conclusión: El equipo APS podría lograr un mayor efecto preventivo post-catástrofe trabajando con otros sectores que incluyan la dimensión salud mental en el abordaje de los problemas sociales críticos en situaciones de desastres, y reservando el trabajo preventivo directo para las personas con alta vulnerabilidad psicológica.
\end{abstract}

Palabras-clave: salud mental; atención primaria de salud; desastres.

\begin{abstract}
Background: There is a high prevalence of emotional disturbances in the population exposed to disasters, and primary health care centers (PHC) could contribute to their prevention. Objective: To analyze post-catastrophe associations between PHC variables and population mental health variables. Method: Study conducted in Chile one year after an $8.8^{\circ}$ Richter earthquake. We applied a questionnaire on the intensity of mental health actions in 16 PHC centers, and scales GHQ-12 and SF-36 for psychological distress and quality of life, in a sample based on households and consultants of the PHCs. We used multiple linear regression analysis for the two samples and the two instruments. Results: The variables on PHC intensity of actions showed no association with the GHQ-12 or SF-36 scores in both samples. The main variables significantly associated with higher scores on GHQ-12 and lower scores on SF-36 were female gender, fewer years of schooling, negative economic situation, less social support and physical damages caused by the earthquake. Conclusion: The PHC teams could achieve greater preventive post-disaster outcome working with other sectors, including mental health dimensions, while addressing the critical disaster social issues, and focusing direct preventive work on those psychologically vulnerable.
\end{abstract}

Keywords: mental health; primary health care; disasters.

${ }^{1}$ Unidad de Salud Mental, Instituto de Salud Poblacional, Facultad de Medicina, Universidad de Chile, Santiago, Chile.

${ }^{2}$ Universidad de Talca, Talca, Chile.

${ }^{3}$ Servicio de Psiquiatría, Hospital de Curicó, Curicó, Chile.

${ }^{4}$ Programa Salud y Comunidad, Instituto de Salud Poblacional, Facultad de Medicina, Universidad de Chile, Santiago, Chile.

Trabajo realizado en los centros de APS y la población de la Región del Maule, Chile.

Correspondencia: Alberto Minoletti, Av. Independencia, 939, Independencia, 8380453 - Santiago, Chile - E-mail: aminoletti2@gmail.com

Apoyo financiero: Fondo Nacional de Investigación y Desarrollo en Salud del Gobierno de Chile (FONIS SA12I2073).

Conflicto de intereses: nada que declarar.

Este es un artículo publicado en acceso abierto (Open Access) bajo la licencia Creative Commons Attribution, que permite su uso, distribución y reproducción en cualquier medio, sin restricciones siempre que el trabajo original sea debidamente citado. 


\section{INTRODUCCIÓN}

La magnitud de los efectos de los desastres naturales en la salud mental ha llevado a considerarlos un problema de salud pública tan prioritario como la salud física de las víctimas ${ }^{1-4}$. Los estudios epidemiológicos han demostrado prevalencias de alteraciones emocionales y/o deterioro funcional en 31 a 55\% de la población expuesta en los meses siguientes al desastre, dependiendo del grado y duración de la exposición y del nivel de factores sociales protectores o agravantes, así como de la metodología usada ${ }^{5-9}$. Los efectos pueden variar desde alteraciones emocionales relativamente leves y transitorias, hasta trastornos moderados a severos de larga duración si no son tratados adecuadamente ${ }^{10}$.

La literatura creciente sobre intervenciones preventivas y tratamientos para las complicaciones psicológicas ${ }^{11-16}$, y la experiencia acumulada en distintos países sobre la organización de servicios para víctimas, han permitido disponer de alternativas para abordar la salud mental post-desastre, aunque no existe acuerdo de cuales intervenciones y servicios serían más efectivos en países en desarrollo ${ }^{1-4,17-20}$.

Considerando que el incremento en Chile de recursos y actividades de salud mental en APS podría haber sido un factor protector en los meses posteriores al terremoto y tsunami del 27 de febrero del 2010 (27-F), se planteó como objetivo indagar la posible asociación entre las acciones de los centros de APS y el estado de salud mental de su población adscrita. El sistema público contaba con un plan nacional de salud mental, un programa de salud mental específico para $\mathrm{APS}^{21,22}$ y un plan de salud mental para situaciones de desastres ${ }^{23}$. Posterior al 27-F se reforzaron los recursos de salud mental en las zonas afectadas $^{24}$ y la APS realizó diferentes acciones preventivas y curativas de salud mental, aunque con una intensidad variable entre los distintos centros ${ }^{25-27}$. La hipótesis subyacente es que los centros de APS con una mayor intensidad de acciones de salud mental se asociarían con un menor nivel de malestar psicológico y un mayor nivel de calidad de vida en su población adscrita.

\section{MÉTODO}

Se efectuó un estudio transversal en las ciudades de la VII Región del Maule de Chile (Curicó, Talca, Linares, Constitución y Pelluhue), 18 meses después del 27-F, con un diseño multinivel que contenía dos unidades de análisis: 1) los centros APS, y 2) los beneficiarios del sistema público de salud de 18 a 65 años. Se utilizó la estructura multinivel para valorar la posible asociación entre la intensidad de las acciones realizadas por APS (nivel superior), con el malestar psicológico y la calidad de vida de la población beneficiaria (nivel inferior).

\section{Universo y muestra}

Respecto de los centros de APS, se tomó la totalidad de ellos en todas las ciudades de la Región $(n=16)$. El universo de beneficiarios para los 16 centros de APS corresponde a 319.296 personas. Se utilizaron dos muestras de personas: una muestra poblacional basada en hogares, y una muestra de consultantes en los centros.

El tamaño de la primera muestra (poblacional) se calculó considerando un estudio realizado previamente en Chile y que arrojó una prevalencia de 6 meses de trastornos mentales de $22,6 \%{ }^{28}$. Se estimó un error máximo de $5 \%$ y un nivel de confianza del 95\%, lo que da un valor de 266. Considerando que se trata de un diseño multinivel, se incorporó un tamaño de efecto de 1,61, basado en un ICC de 0,03839, según estudio previo de tratamiento de depresión en APS $^{29}$. Esto implicó aumentar en $61 \%$ la cantidad de casos (428 casos totales y 27 para cada centro). La muestra final se obtuvo con un proceso de selección aleatoria en tres etapas: primero las manzanas, luego los hogares y finalmente las personas (usando el método de Kish). Estuvo constituida por 720 personas, con 53,5\% de mujeres y una edad promedio de 39,7 $\pm 13,7$ años.

La segunda muestra (consultantes por morbilidad general) se calculó con una prevalencia de trastornos mentales de $52,5 \%$, basado en otro estudio realizado en el país ${ }^{30}$, con un error máximo de 5\% y un nivel de confianza del $95 \%$, lo que da un valor de 378. La estimación del tamaño de efecto se hizo de igual forma que en la muestra anterior, obteniéndose un valor de 1,88 (la que aumenta la muestra a 711 casos totales y 45 para cada centro APS). Se realizó un procedimiento de selección aleatoria por conglomerados a partir de las agendas médicas para atención de morbilidad general dentro de cada centro. La muestra obtenida fue de 720 sujetos, con $79,4 \%$ de mujeres y una edad promedio de 42,4 $\pm 12,7$ años.

\section{Instrumentos y cuestionarios}

Para valorar la intensidad de las acciones realizadas por los centros de APS se aplicó un cuestionario elaborado ad hoc, con un proceso de validación de contenido y adaptación semántica, que ya fue descrito en una publicación previa ${ }^{27}$. En la Tabla 1 se resumen las áreas de evaluación y sus resultados.

En las dos muestras de personas se utilizó el Cuestionario de Salud General de Goldberg en su versión de 12 ítems (GHQ-12) para medir malestar psicológico ${ }^{31}$ y el Cuestionario de Salud SF-36 para medir calidad de vida relacionada con la salud ${ }^{32}$. Adicionalmente, se consultó por otras variables relevantes tales como: sexo, edad, situación de pareja, nivel educacional, situación laboral, efectos negativos del desastre, intervenciones de salud mental recibidas y nivel de apoyo social. 
Tabla 1. Cuestionario para evaluar centros de atención primaria: principales áreas, aspectos evaluados e indicadores

\begin{tabular}{|c|c|c|}
\hline Áreas de evaluación & Aspectos evaluados & Indicadores \\
\hline 1. Recursos humanos & Profesionales y técnicos de APS & Horas destinadas a salud mental \\
\hline 2. Accesibilidad geográfica y económica & $\begin{array}{l}\text { Tiempo y costo del traslado del hogar al } \\
\text { centro AP }\end{array}$ & Minutos empleados y valor del transporte \\
\hline 3. Programas de salud mental & $\begin{array}{l}\text { Incidencia tratada y adherencia a } \\
\text { tratamiento de depresión y trastorno ansioso }\end{array}$ & $\begin{array}{l}\text { Tasas de ingreso en los } 6 \\
\text { meses posteriores al terremoto } \\
\text { (por 10,000 de población > } 15 \text { años). } \\
\text { Promedios de meses de adherencia }\end{array}$ \\
\hline \multirow[t]{4}{*}{$\begin{array}{l}\text { 4. Intensidad de acciones de salud mental en } \\
\text { los meses siguientes al terremoto }\end{array}$} & $\begin{array}{l}\text { Preparación en salud mental para } \\
\text { situaciones de desastre }\end{array}$ & $\begin{array}{l}\text { Conocimiento de planes nacionales y } \\
\text { comunales, y preparación para enfrentar } \\
\text { desastres }\end{array}$ \\
\hline & Acciones comunitarias de salud mental & $\begin{array}{l}\text { Cobertura poblacional y duración de trabajo } \\
\text { con líderes, apoyo emocional de adultos y } \\
\text { actividades lúdicas con niños }\end{array}$ \\
\hline & Coordinación intra e intersectorial & $\begin{array}{l}\text { Diversidad de instituciones y organizaciones } \\
\text { contactadas de salud y de otros sectores }\end{array}$ \\
\hline & Cuidado de la salud mental del personal APS & $\begin{array}{l}\text { Cobertura del personal con actividades } \\
\text { educativas y de apoyo emocional }\end{array}$ \\
\hline
\end{tabular}

\section{Trabajo de campo}

Los encuestadores fueron profesionales recién egresados, que recibieron un entrenamiento para este trabajo de dos días de duración. Previo a la entrevista se obtuvo el consentimiento informado de las personas, manteniéndose la confidencialidad de los datos entregados. Todo el trabajo de campo se realizó entre julio y octubre del año 2011.

El proyecto de investigación y los consentimientos informados utilizados fueron aprobados por el Comité de Ética de la Facultad de Medicina, de la Universidad de Chile ( ${ }^{\circ}$ 051-2010).

\section{Análisis estadísticos}

Para valorar nuestras hipótesis se efectuaron análisis de regresión lineal múltiple que incorporaban la estructura multinivel, para cada una de las dos poblaciones y para cada uno de los dos resultados evaluados (puntaje en el GHQ-12 y puntaje en el SF-36). En cada caso se construyeron modelos de efectos principales, que incorporaron las 12 variables a nivel del centro de APS (nivel superior) y 15 variables independientes a nivel de cada individuo (nivel inferior). Se realizó un procedimiento de eliminación progresiva de las variables que no eran significativas, de tipo backward, en forma manual, hasta obtener el modelo parsimonioso.

\section{RESULTADOS}

De las 4 áreas de acciones de salud mental realizadas por los centros APS en relación al desastre, "la cobertura del personal APS con educación y apoyo emocional después del desastre" fue la que tuvo el nivel de cumplimiento más alto (mediana de los 16 centros de $81,9 \%$ de un máximo de $100 \%$ ), mientras que "el conocimiento de planes de salud mental y de desastres y la preparación para enfrentar desastres previamente" fue la que tuvo la evaluación más baja, con una mediana de cumplimiento de $31,9 \%$ (Tabla 2).

Las dos muestras de beneficiarios mostraron diferencias (Tabla 3), caracterizándose los consultantes por morbilidad, en comparación con la muestra poblacional, por tener una mayor proporción de sexo femenino $(79,4 \%)$, más edad (media de 42,4 años), menos años de estudio (media de 9,2 años), menor porcentaje de personas con trabajo regular $(29,2 \%)$ y menor ingreso familiar mensual $(\$ 72.370,34)$. Poco más del $60 \%$ de las personas en ambas muestras sufrió uno o más efectos negativos por el desastre. A su vez, los consultantes tienen un puntaje más bajo en la escala de apoyo social y un menor nivel de salud mental, expresado por mayor malestar psicológico en el GHQ-12 y menor calidad de vida en el SF-36.

En relación al análisis multi-nivel para malestar psicológico, en ambas muestras se observó mayor variabilidad intra centro APS que entre centros, por lo que los puntajes de los individuos de un mismo centro casi no tienen correlación entre ellos (ICC $<0.01)$. Ninguna de las variables correspondientes a la intensidad de las acciones de salud mental mostró relación con el puntaje en el GHQ-12. Las variables que en ambas muestras se asociaron significativamente a un mayor puntaje en el GHQ-12 fueron ser mujer, tener menos años de estudio y tener menor apoyo social; en la muestra de consultantes a APS se agrega además el tener más edad, haber sufrido algún daño en la vivienda producto del desastre y haber estado en vivienda de emergencia o allegado por más de 14 días; y en la muestra basada en viviendas se agrega haber sufrido personalmente algún daño físico moderado o grave producto del desastre o que algún familiar lo haya sufrido, y que el centro APS dedique un menor porcentaje de horas profesionales a salud mental (Tabla 4). 
Tabla 2. Intensidad de las acciones de salud mental realizadas por los centros de Atención Primaria de Salud (APS) con relación al terremoto/ maremoto. VII Región del Maule de Chile, 2011.

\begin{tabular}{lccc} 
& Media $^{*}$ & $\begin{array}{c}\text { Desviación } \\
\text { estándar }\end{array}$ & Mediana $^{*}$ \\
Conocimiento de planes y preparación para enfrentar desastres & $35,0 \%$ & 18,5 & $31,9 \%$ \\
Cobertura poblacional y duración de acciones comunitarias preventivas & $39,9 \%$ & 24,8 & $39,6 \%$ \\
Diversidad de instituciones y organizaciones con coordinación & $60,9 \%$ & 20,9 & $56,3 \%$ \\
Cobertura del personal APS con educación y apoyo emocional & $68,4 \%$ & 31,8 & $81,9 \%$ \\
\hline
\end{tabular}

${ }^{\star}$ Media y mediana de los porcentajes de cumplimiento de los 16 centros APS en relación con un estándar definido por grupo de expertos

Tabla 3. Características de las 2 poblacionales de beneficiarios de centros Atención Primaria de Salud (APS) estudiadas: muestra de población general basada en hogares y muestra de consultantes por morbilidad general en APS. VII Región del Maule de Chile, 2011.

\begin{tabular}{lcc} 
& Consultantes a APS & 720 \\
$\mathrm{~N}$ & Población general & 432 \\
Sexo femenino (\%) & $52,1 \%$ & $79,4 \%$ \\
Edad (media en años) & 39,72 & 42,43 \\
Casado(a) o vive con pareja (\%) & $57,0 \%$ & $61,0 \%$ \\
Años de estudio (media en años) & 10,95 & 9,1 \\
Trabajo regular (\%) & $48,1 \%$ & $29,2 \%$ \\
Daño a vivienda superior a 25\% (\%) & $18,7 \%$ & $18,6 \%$ \\
Allegado o en vivienda de emergencia por más de 14 días (\%) & $15,5 \%$ & $18,6 \%$ \\
Daño físico moderado a grave (\%) & $0,9 \%$ & $2,8 \%$ \\
Familiar o amigo, con daño físico, muerte o desaparición por más de 1 hr. (\%) & $17,1 \%$ & $18,1 \%$ \\
Sin trabajo por más de 14 días y sin compensación total (\%) & $32,6 \%$ & $25,4 \%$ \\
Uno o más efectos negativos por el terremoto o maremoto (\%) & $63,2 \%$ & $60,7 \%$ \\
GHQ-12 (media de puntaje) & 22,62 & 24,99 \\
SF-36 (media de puntaje) & 66,54 & 51,21 \\
Escala apoyo social (media) & 19,26 & 18,50 \\
Ingreso familiar mensual (pesos) & $\$ 333795,82$ & $\$ 72370,34$ \\
\hline
\end{tabular}

Tabla 4. Resultados del análisis multi-nivel* para GHQ-12 en las 2 muestras estudiadas de beneficiarios de Atención Primaria de Salud (APS): consultantes a APS por morbilidad y muestra de población general basada en hogares. VII Región del Maule de Chile, 2011.

\begin{tabular}{|c|c|c|c|c|}
\hline \multirow{2}{*}{ Variables con asociación significativa } & \multicolumn{2}{|c|}{ Consultantes APS } & \multicolumn{2}{|c|}{ Población general } \\
\hline & $\mathbf{z}$ & $\mathbf{P}>|\mathbf{z}|$ & $\mathbf{z}$ & $\mathbf{P}>|\mathbf{z}|$ \\
\hline Edad & 2,08 & 0,04 & s.a.s. & s.a.s. \\
\hline Sexo & 2,48 & 0,01 & $-3,92$ & $<0,01$ \\
\hline Años de estudio & $-3,68$ & $<0,01$ & $-4,46$ & $<0,01$ \\
\hline Daño físico en encuestado (moderado o grave) & s.a.s. & s.a.s. & 2,83 & 0,01 \\
\hline Daño en familiar o amigo & s.a.s. & s.a.s. & 1,98 & 0,05 \\
\hline Daño de la vivienda & 2,19 & 0,03 & s.a.s. & s.a.s. \\
\hline Estuvo en vivienda de emergencia o allegado por más de 14 días & 2,71 & 0,01 & s.a.s. & s.a.s. \\
\hline Puntaje Escala de Apoyo Social & $-6,54$ & $<0,01$ & $-3,37$ & $<0,01$ \\
\hline $\begin{array}{l}\text { \% del total de horas de profesionales del Centro de APS que se } \\
\text { dedican a salud mental }\end{array}$ & s.a.s. & s.a.s. & $-1,97$ & 0,05 \\
\hline
\end{tabular}

${ }^{*}$ modelo parsimonioso; s.a.s. $=$ sin asociación significativa

Al igual que para malestar psicológico, la calidad de vida en las dos muestras mostró mayor variabilidad intra centro APS que entre centros y ninguna de las variables correspondientes a las acciones de los centros APS tuvo relación con el SF - 36. La mayoría de las variables que mostraron asociación con el GHQ-12 también se asociaron con un menor puntaje en el SF-36 (con excepción de daño físico en el encuestado producido por el desastre y porcentaje de horas que los profesionales de APS destinan a salud mental). Además, con el SF-36 aparece asociación significativa con variables económicas que no se observaron con el GHQ-12: en ambas muestras la menor calidad de vida se asocia con menor accesibilidad económica al centro (mayor cantidad de dinero que las personas gastan en trasladarse al centro), en la muestra poblacional la asociación es también con menor ingreso mensual del grupo familiar y en la muestra de consultantes la asociación es con mayor porcentaje de población en situación de pobreza en el lugar donde viven (Tabla 5). 
Tabla 5. Resultados del análisis multi-nivel* para SF-36 en las 2 muestras estudiadas de beneficiarios de Atención Primaria de Salud (APS): consultantes a APS por morbilidad y muestra de población general basada en hogares. VII Región del Maule de Chile, 2011.

\begin{tabular}{|c|c|c|c|c|}
\hline \multirow{2}{*}{ Variables con asociación significativa } & \multicolumn{2}{|c|}{ Consultantes APS } & \multicolumn{2}{|c|}{ Población general } \\
\hline & $\mathbf{z}$ & $\mathbf{P}>|\mathbf{z}|$ & $\mathbf{z}$ & $\mathbf{P}>|\mathbf{z}|$ \\
\hline Edad & $-4,64$ & $<0,01$ & $-4,79$ & $<0,01$ \\
\hline Sexo & 3,28 & $<0,01$ & $-5,12$ & $<0,01$ \\
\hline Años de estudio & 3,14 & $<0,01$ & s.a.s. & s.a.s. \\
\hline Ingresos mensuales del grupo familiar & s.a.s. & s.a.s. & 3,37 & $<0,01$ \\
\hline Porcentaje de pobreza en la población adscrita & $-1,78$ & 0,08 & s.a.s. & s.a.s. \\
\hline Daño en familiar o amigo & $-2,36$ & 0,02 & $-2,28$ & 0,02 \\
\hline Daño de la vivienda & $-2,02$ & 0,04 & s.a.s. & s.a.s. \\
\hline Estuvo en vivienda de emergencia o allegado por más de 14 días & $-2,22$ & 0,03 & s.a.s. & s.a.s. \\
\hline Puntaje Escala de Apoyo Social & 2,16 & 0,03 & s.a.s. & s.a.s. \\
\hline Accesibilidad económica a centro APS & $-2,48$ & 0,01 & $-2,40$ & 0,02 \\
\hline $\begin{array}{l}\text { Horas totales de profesionales (médicos y no médicos) por } 10.000 \\
\text { población inscrita }\end{array}$ & $-2,33$ & 0,02 & s.a.s. & s.a.s. \\
\hline
\end{tabular}

${ }^{*}$ modelo parsimonioso; s.a.s. $=$ sin asociación significativa

\section{DISCUSIÓN}

Los resultados del presente estudio no corroboraron la hipótesis planteada, de que los centros APS con una mayor intensidad de acciones de salud mental se asociarían con un menor nivel de malestar psicológico y un mayor nivel de calidad de vida en su población adscrita. Una posible explicación para este fenómeno puede corresponder a que la frecuencia, duración y cobertura de dichas acciones hayan sido insuficientes para la magnitud del desastre y sus consecuencias en la salud mental, tal como se describió en un artículo anterior ${ }^{27}$. Al mismo tiempo, es necesario considerar que a pesar de los avances que ha tenido la APS y su programa de salud mental, aún existen barreras estructurales importantes para el logro de una atención de alta cobertura y calidad. Ejemplos de barreras detectadas en el presente estudio son la menor accesibilidad económica (definida como costo de movilización para trasladarse de la casa al centro APS) y la baja cantidad de recursos humanos de APS (ya sean específicos para salud mental o para equipos de APS en general), las cuáles mostraron asociación con mayor malestar psicológico y menor calidad de vida.

Una explicación alternativa puede relacionarse con los tipos de intervenciones realizadas, las cuáles si bien tuvieron un alto valor humanitario para las víctimas del desastre, puede que no hayan sido las de mayor efectividad para el tipo de problemas de salud mental que experimentaron.

Desde una perspectiva más amplia, no resultaría ilógico considerar que un conjunto de determinantes sociales de la salud mental pueda adquirir una preponderancia mayor en situaciones de desastres, profundizando la vulnerabilidad de algunos grupos poblacionales, tal como ha sido demostrado por algunos autores ${ }^{33}$. De esta manera, el impacto de las intervenciones de APS podría disminuirse si ellas no se realizan en conjunto con otros programas sociales que respondan a las necesidades críticas de las víctimas de la catástrofe. Concordantemente, los resultados mostraron una relación significativa de variables asociadas al nivel socioeconómico (menor número de años de estudio, menor ingreso familiar y mayor porcentaje de pobreza en la población adscrita) y al género (ser mujer), con variables negativas de salud mental (mayor malestar psicológico y menor calidad de vida).

Este estudio también ha mostrado, tal como era esperable, que las variables asociadas a la vivienda son particularmente importantes en situaciones de desastre, existiendo una asociación entre haber sufrido algún daño en la vivienda y tener un menor nivel de salud mental (en la muestra de consultantes); similar asociación existe con haber estado en vivienda de emergencia o allegado por más de 14 días. Resulta razonable pensar, por consiguiente, que las acciones de salud mental de APS podrían disminuir su efectividad si las personas continúan experimentando estrés y situaciones de re-traumatización que implican el no tener un hogar propio y vivir de allegadas o en viviendas de emergencia ${ }^{34}$.

Otro resultado importante es la asociación significativa entre el nivel de apoyo social y las dos variables de salud mental estudiadas. El apoyo social es un componente crítico del capital social y existe evidencia de intervenciones efectivas para reforzarlo y así mejorar el nivel de salud mental de una comunidad $^{35}$. De esta manera, las políticas públicas debieran considerar estrategias en este sentido, tanto en forma genérica, para contribuir a la promoción de la salud mental, como en forma específica, para enfrentar de mejor manera los desafíos de las situaciones de desastres.

Este estudio también invita a reflexionar sobre el rol del equipo APS en salud mental en este tipo de catástrofes y sobre el tipo de intervenciones susceptibles de tener un impacto en estas situaciones. Posiblemente, APS puede lograr un mayor 
efecto preventivo en forma indirecta, trabajando con otros sectores para que incluyan la dimensión salud mental en el abordaje de los problemas sociales críticos en situaciones de desastres, y reservando el trabajo preventivo directo para las personas con alta vulnerabilidad psicológica (ej. personas en tratamiento por enfermedades mentales y personas con crisis emocionales post-desastre), tal como ha sido propuesto por algunos autores ${ }^{33}$.

El presente estudio tiene algunas limitantes propias de la naturaleza impredecible del fenómeno que aborda. En el momento que ocurre un desastre no se dispone de recursos para la evaluación de servicios sanitarios ni es posible solicitarlos con antelación. No es posible descartar totalmente que la medición retrospectiva de las acciones de salud mental y la ausencia de registros adecuados en APS (con mayores limitaciones después del desastre) puedan haber provocado alguna distorsión en los resultados obtenidos. No obstante, las limitaciones descritas, esta primera evaluación en Chile de acciones de salud mental post-desastre y su asociación con el nivel de salud mental demuestra que este tipo de estudios es factible y que entregan información útil para mejorar respuestas a necesidades de la población.

Considerando la frecuencia de los desastres naturales en países latinoamericanos, las políticas de salud mental deberían contemplar la formación de equipos APS en intervenciones protocolizadas basadas en evidencias, junto con acciones intersectoriales que aborden los determinantes sociales. Concomitantemente, se requiere disponer de un sistema de evaluación para aplicación en condiciones de desastre, de modo de poder caracterizar la población afectada, las intervenciones de salud mental realizadas y sus impactos, generando evidencias para enfrentar de mejor manera este problema de salud pública.

\section{REFERENCIAS}

1. World Health Organization. Mental health in emergencies. Geneva: WHO; 2003.

2. Rodríguez J, Zaccarelli M, Pérez R, editors. Guía práctica de salud mental en situaciones de desastres. Washington: Organización Panamericana de la Salud; 2006.

3. Van Ommeren M, Saxena S, Saraceno B. Mental and social health during and after acute emergencies: emerging consensus. Bull World Health Organ. 2005;83(1):71-5, discussion 75-6. PMid:15682252.

4. Inter-Agency Standing Comité. Guía del IASC sobre salud mental y apoyo psicosocial en emergencias humanitarias y catástrofes. Ginebra: Naciones Unidas; 2007

5. Lima BR, Santacruz H, Lozano J, Luna J, Pai S. La atención primaria de salud mental en las víctimas del desastre de Armero, Colombia. Acta Psiquiatr Psicol Am Lat. 1988;34(1):13-32. PMid:3188963.

6. Lima BR, Chavez H, Samaniego N, Pompei MS, Pai S, Santacruz H, et al. Disaster severity and emotional disturbance: implications for primary mental health care in developing countries. Acta Psiquiatr Scand. 1989;79(1):74-82. http://dx.doi.org/10.1111/j.1600-0447.1989.tb09236.x. PMid:2929384.

7. North CS, Nixon SJ, Shariat S, Mallonee S, McMillen JC, Spitznagel EL, et al. Psychiatric disorders among survivors of the Oklahoma City bombing. JAMA. 1999;282(8):755-62. http://dx.doi.org/10.1001/jama.282.8.755. PMid:10463711.

8. Wang PS, Gruber MJ, Powers RE, Schoenbaum M, Speier AH, Wells $\mathrm{KB}$, et al. Mental health service use among Hurricane Katrina survivors in the eight months after the disaster. Psychiatr Serv. 2007;58(11):1403-11. http://dx.doi.org/10.1176/ps.2007.58.11.1403. PMid:17978249.

9. Abramson D, Stehling-Ariza T, Garfield R, Redlener I. Prevalence and predictors of mental health distress post-Katrina: findings from the Gulf Coast. Child and family health study. Disaster Med Public Health Prep. 2008;2(2):77-86. http://dx.doi.org/10.1097/DMP.0b013e318173a8e7. PMid:18520693.
10. Figueroa RA, Marín H, González M. Apoyo psicológico en desastres: propuesta de un modelo de atención basado en revisiones sistemáticas y metaanálisis. Rev Med Chil. 2010;138(2):143-51. http://dx.doi.org/10.4067/ S0034-98872010000200001. PMid:20461301.

11. Stein DJ, Ipser JC, Seedat S. Pharmacotherapy for post traumatic stress disorder (PTSD). Cochrane Database Syst Rev. 2006;(1):CD002795. PMid:16437445.

12. Rose SC, Bisson J, Churchill R, Wessely S. Psychological debriefing for preventing post traumatic stress disorder (PTSD). Cochrane Database Syst Rev. 2002;(2):CD000560. PMid:12076399.

13. Bisson J, Andrew M. Psychological treatment of post-traumatic stress disorder (PTSD). Cochrane Database Syst Rev. 2007;(3):CD003388. PMid:17636720.

14. Roberts NP, Kitchiner NJ, Kenardy J, Bisson JI. Multiple session early psychological interventions for the prevention of post-traumatic stress disorder. Cochrane Database Syst Rev. 2009;(3):CD006869. PMid:19588408.

15. Roberts NP, Kitchiner NJ, Kenardy J, Bisson JI. Early psychological interventions to treat acute traumatic stress symptoms. Cochrane Database Syst Rev. 2010;(3):CD007944. PMid:20238359.

16. Shalev AY, Ankri Y, Israeli-Shalev Y, Peleg T, Adessky R, Freedman S. Prevention of posttraumatic stress disorder by early treatment: Results from the Jerusalem Trauma Outreach and Prevention Study. Arch Gen Psychiatry. 2012;69(2):166-76. http://dx.doi.org/10.1001/archgenpsychiatry.2011.127. PMid:21969418.

17. Prasetiyawan, Viora E, Maramis A, Keliat BA. Mental health model of care programmes after the tsunami in Aceh, Indonesia. Int Rev Psychiatry. 2006;18(6):559-62. http://dx.doi.org/10.1080/09540260601039959. PMid:17162697.

18. Kim Y, Abe Y, Araki H, Fujita M, Iwai K, Kato H, et al. Guideline for local mental health care activities after a disaster [Internet]. Tokyo: National Institute of Mental Health, National Center of Psychiatry and Neurology; 2016 [citado 15 feb 2016]. Disponible en: http://www.ncnp.go.jp/nimh/ seijin/EnglishDisasterMentalHealth.pdf 
19. Organización Mundial de la Salud. Primera ayuda psicológica: guía para trabajadores de campo. Ginebra: Organización Mundial de la Salud; 2012.

20. Bassilios B, Reifels L, Pirkis J. Enhanced primary mental health services in response to disaster. Psychiatr Serv. 2012;63(9):868-74. http://dx.doi. org/10.1176/appi.ps.201100534. PMid:22707018.

21. Minoletti A, Sepúlveda R, Horvitz-Lennon M. Twenty years of mental health policies in Chile: Lessons and challenges. Int J Ment Health. 2012;41(1):2137. http://dx.doi.org/10.2753/IMH0020-7411410102.

22. Minoletti A, Rojas G, Horvitz-Lennon M. Salud Mental en Atención Primaria en Chile: aprendizajes para Latinoamérica. Cad Saude Colet. 2012;20(4):440-7. http://dx.doi.org/10.1590/S1414-462X2012000400006.

23. Chile. Ministerio de Salud. Plan de Protección de la Salud Mental en Situaciones de Emergencias y Desastres. Santiago: Departamento de Salud Mental, Subsecretaría de Salud Pública; 2008.

24. Erazo C, Pemjean A. Red de atención de salud mental en Chile y el terremoto de febrero 2010: fortalezas, daños y respuestas. Rev Chil Salud Pública. 2010;14(1):59-65.

25. Organización Panamericana de la Salud. Protección de la salud mental luego del terremoto y tsunami del 27 de febrero de 2010 en Chile: crónica de una experiencia. Santiago: Organización Panamericana de la Salud; 2010.

26. Minoletti A, Grandón P, Jiménez A, Saldivia S. Estrategias de intervención en salud mental post terremoto y tsunami de Chile 2010: aprendizajes y desafíos desde la atención primaria. Cuad Psiquiatr Comunitaria. 2010;10(1):35-47.

27. Vitriol A, Minoletti A, Alvarado A, Sierralta P, Cancino A. Respuesta de los centros de atención primaria en salud mental después del terremoto y tsunami del 2010 en la región del Maule. Rev Med Chil. 2014;142(9):1120-7. http://dx.doi.org/10.4067/S0034-98872014000900005. PMid:25517051.
28. Vicente B, Rioseco P, Saldivia S, Kohn R, Torres S. Estudio chileno de prevalencia de patología psiquiátrica (DSM III - R / CIDI) (ECPP). Rev Med Chil. 2002;130(5):527-36. http://dx.doi.org/10.4067/S003498872002000500007. PMid:12143273.

29. Alvarado R, Rojas G, Minoletti A, Alvarado F, Domínguez C. Depression program in primary health care: the Chilean experience. Int J Ment Health. 2012;41(2):38-47. http://dx.doi.org/10.2753/IMH0020-7411410103.

30. Goldberg DP, Lecubrier Y. Form and frequency of mental disorders across centres. In: Üstün TB, Sartorius N, editors. Mental illness in general health care: an international study. Chichester: John Wiley \& Sons; 1995.

31. Goldberg DP, Wiliams P. Cuestionario de Salud General, (GHQ) Guía para el usuario de las distintas versiones. Barcelona: Masson; 1996.

32. Vilagut G, Ferrer M, Rajmil L, Rebollo P, Permanyer-Miralda G, Quintana J, et al. El Cuestionario de Salud SF-36 Español: una década de experiencia y nuevos desarrollos. Gac Sanit. 2005;19(2):135-50. http://dx.doi. org/10.1157/13074369. PMid:15860162.

33. Norris FH, Friedman MJ, Watson PJ. 60,000 disaster victims speak: part II. Summary and implications of the disaster mental health research. Psychiatry. 2002;65(3):240-60. http://dx.doi.org/10.1521/psyc.65.3.240.20169. PMid:12405080.

34. Galea S, Tracy M, Norris F, Coffey SF. Financial and social circumstances and the incidence and course of PTSD in Mississippi during the first two years after Hurricane Katrina. J Trauma Stress. 2008;21(4):357-68. http:// dx.doi.org/10.1002/jts.20355. PMid:18720399.

35. Jenkins R, Minoletti A. Promoting mental health: a crucial component of all public policy. In: Leppo K, Ollila E, Peña S, Wismar M, Cook S, editors. Health in all policies. Helsinki: Ministry of Social Affairs and Health; 2013.

Recibido en: Marzo 19, 2018 Aprobado en: Jul. 24, 2018 